\title{
Caracterización de las metodologías de investigación en finanzas bursátiles en Colombia del 2015 al 2020
}

\author{
Paula Andrea Navarro Pérez*
}

Recibido: 21 de septiembre de 2020

Aprobado: 06 de noviembre de 2020

\begin{abstract}
Navarro, P. A. (2020). Caracterización de las metodologías de investigación en finanzas bursátiles en Colombia del 2015 al 2020. Revista Activos, 18(2), 205-222. https://doi.org/10.15332/25005278/6284
\end{abstract}

\section{Clasificación JEL: O16, G1, G2}

\section{Resumen}

El objetivo de este documento es caracterizar las metodologías de investigación utilizadas por los artículos en finanzas bursátiles entre los años 2015 y 2020 en Colombia, mostrando cuáles han sido los enfoques, el alcance, el diseño y los métodos de recolección de información empleados por

\footnotetext{
* Contadora pública por la Universidad Nacional de Colombia; magíster en Business Administration por la Universidad Purdue, estudiante de doctorado en Administración en la Universidad Nacional de Colombia. Profesora asistente en la Fundación Universitaria Los Libertadores, miembro del grupo de investigación Reflexión Económica, Administrativa y Contable.

Correo electrónico: panavarrop@libertadores.edu.co ORCID: https://orcid.org/0000-0001-7253-6646
} 
los autores; todo esto, a través de una revisión de literatura que aplica un enfoque de investigación cualitativo con alcance descriptivo. De manera general, el enfoque cuantitativo predomina en las investigaciones financieras bursátiles colombianas durante el periodo de estudio. Además, dicho enfoque, en la mayoría de las veces, se combina con un alcance correlacional o explicativo, con un diseño metodológico longitudinal de corte no experimental o de corte transversal y con métodos de recolección de información basados en indicadores o pruebas estandarizadas.

Palabras clave: finanzas bursátiles, metodologías de investigación en finanzas, investigación financiera, revisión de literatura en finanzas.

\title{
Characterization of research methodologies in stock market finance in Colombia from 2015 to 2020
}

\begin{abstract}
This article aims to characterize the research methodologies used by articles in stock market finance between 2015 and 2020 in Colombia, showing what have been the approaches, scope, design, and data collection methods used by the authors, all this, through a literature review that applies a qualitative research approach with descriptive scope. Overall, the quantitative research approach predominates in the Colombian financial stock market research during the study period. In addition, this approach, in most cases, is combined with a correlational or explanatory scope, with a non-experimental or cross-sectional a longitudinal methodological design, and with data collection methods based on indicators or standardized tests.
\end{abstract}

Keywords: stock market finance, finance research methodologies, financial research, finance literature review. 


\section{Introducción}

La investigación en finanzas bursátiles en Colombia cobró importancia desde finales del siglo xx cuando se empezaron a dinamizar las transacciones bursátiles como parte de los cambios económicos surgidos a raíz de la apertura y del movimiento de flujos de capital con otros países (Cárdenas y Rojas, 1996). No obstante, dichas investigaciones eran de carácter esporádico, se impulsaban principalmente por organismos gubernamentales y únicamente se consolidaron como un área de interés formal en la academia a principios del siglo XXI cuando, mediante la fusión de las bolsas de valores de Bogotá, Medellín y Cali, se creó la Bolsa de Valores de Colombia, cuya función principal se enfocó en canalizar instrumentos de financiación suficientes para el aparato productivo del país.

Si bien el tema de las finanzas bursátiles se considera relativamente nuevo en Colombia, los avances que se han hecho en materia investigativa han sido significativos, puesto que, en estas dos últimas décadas, el surgimiento de trabajos académicos en este ámbito ha cobrado relevancia y hoy en día, se considera en auge. Inicialmente, dichos trabajos se enfocaron en caracterizar las dinámicas básicas del mercado bursátil colombiano, indicando el funcionamiento de los principales títulos valores que allí se negocian (Atehortúa Granados, 2012; Uribe Gil, 2007).

Más recientemente, las investigaciones se han encaminado hacia la explicación de fenómenos más específicos y complejos que subyacen en el mercado bursátil colombiano, aplicando modelos estadísticos y econométricos que han surgido en la academia, en el marco del análisis de portafolios, de eficiencia de mercados, de medición de riesgo, entre otros.

Ahora bien, hoy por hoy existen pocos trabajos académicos que muestren el estado actual de este campo, caractericen las metodologías de investigación que se han aplicado y planteen las expectativas para estudios futuros. Debido a eso, el objetivo principal de este trabajo es caracterizar las metodologías de investigación utilizadas por los artículos en finanzas bursátiles entre los años 2015 y 2020, mostrando cuáles han sido los 
enfoques, el alcance, el diseño y los métodos de recolección de información empleados por los autores.

Para el cumplimiento de ese objetivo, este trabajo se estructura en tres secciones: en la primera, se describe la metodología utilizada -la cual es de corte cualitativo con alcance descriptivo- y se lleva a cabo una revisión de literatura; en la segunda, se muestran los resultados obtenidos de dicha revisión y se plantea una discusión en torno a ellos; finalmente, en la tercera, se presentan las conclusiones de este estudio.

\section{Metodología}

Para el diseño metodológico de este trabajo se utiliza un enfoque cualitativo con alcance descriptivo que permita (por medio de una revisión de literatura) identificar y caracterizar los procesos de investigación que se han llevado a cabo en Colombia en el ámbito de las finanzas bursátiles. Para delimitar el alcance de dicha revisión, se acotó el universo documental a aquellos artículos publicados entre el año 2015 y el 2020 en revistas colombianas indexadas por el Ministerio de Ciencia Tecnología e Innovación (Minciencias) con corte a junio 2020. Se utiliza esta indexación debido a que es una de las más significativas a nivel nacional y abarca revistas que reciben artículos no solo de Colombia, sino de otros países en Latinoamérica y en el resto del mundo, tanto en español, como en inglés o portugués. Consecuentemente, se encontró que 21 revistas trabajan temas financieros (que a su vez pueden estar dentro de líneas contables, administrativas o económicas). En la tabla 1 , se presentan las revistas seleccionadas para la revisión con su respectiva categorización a junio de 2020. 
Tabla 1. Revistas colombianas indexadas Minciencias con temas financieros

\begin{tabular}{|c|c|}
\hline Revista & Categoría \\
\hline AD-minister & B \\
\hline Aglala & B \\
\hline Aibi & B \\
\hline Dimensión empresarial & $\mathrm{B}$ \\
\hline Cuadernos de Contabilidad (Universidad Javeriana) & B \\
\hline Cuadernos de Administración (Universidad del Valle) & B \\
\hline Estudios Gerenciales & B \\
\hline Finanzas y Política Económica & $\mathrm{B}$ \\
\hline Pensamiento y Gestión & B \\
\hline Investigación y Reflexión & B \\
\hline Revista Escuela de Administración de Negocios & B \\
\hline Suma de Negocios & $\mathrm{B}$ \\
\hline Cuadernos de Administración (Universidad Javeriana) & $\mathrm{C}$ \\
\hline Criterio Libre & $\mathrm{C}$ \\
\hline Contaduría Universidad de Antioquia & $\mathrm{C}$ \\
\hline Clio América & $\mathrm{C}$ \\
\hline Económicas CuC & $\mathrm{C}$ \\
\hline INNOVAR & $\mathrm{C}$ \\
\hline Semestre Económico & $\mathrm{C}$ \\
\hline Universidad y Empresa & $\mathrm{C}$ \\
\hline Tendencias & $\mathrm{C}$ \\
\hline
\end{tabular}

Fuente: elaboración propia.

Nota: no se encontraron revistas que trabajen temas financieros en categoría A1 o A2. 
Posteriormente, se construyó una matriz de recolección de información revisando uno a uno los números publicados por las revistas seleccionadas para el periodo de estudio. Para el diligenciamiento de dicha matriz se tuvo en cuenta, primero, aspectos de identificación del artículo como el título, el año de publicación, la revista en la cual fue publicado y el tipo de artículo; y segundo, elementos para caracterizar la metodología de investigación utilizada. Así, a partir de lo propuesto por Hernández Sampieri, Fernández Collado y Baptista Lucio (2014), se identificó: (1) el enfoque de investigación, (2) el alcance de la metodología, (3) el diseño metodológico, y (4) los instrumentos de recolección de información.

En la tabla 2 se resumen los elementos de identificación y caracterización de la matriz construida para la revisión documental.

Tabla 2. Matriz de identificación y caracterización de los artículos

\begin{tabular}{|c|c|c|c|}
\hline \multicolumn{4}{|c|}{ 1. Identificación } \\
\hline \multicolumn{4}{|l|}{ Año } \\
\hline \multicolumn{4}{|l|}{ Título } \\
\hline \multicolumn{4}{|l|}{ Revista } \\
\hline \multicolumn{4}{|c|}{ 2. Caracterización de la metodología ${ }^{\star}$} \\
\hline a. Enfoque & b. Alcance & c. Diseño & d. Instrumentos \\
\hline Cualitativo & $\mathrm{N} / \mathrm{A}^{* *}$ & $\begin{array}{l}\text { - Etnográfico } \\
\text { - Teoría } \\
\text { fundamentada } \\
\text { - Narrativo } \\
\text { - Fenomenológico } \\
\text { - Investigación-acción } \\
\text { - Estudio de caso }\end{array}$ & $\begin{array}{l}\text { - Bitácoras o anotaciones } \\
\text { - Observación } \\
\text { - Entrevistas } \\
\text { - Grupos focales } \\
\text { - Documentos y registros } \\
\text { - Biografías e historias } \\
\text { de vida }\end{array}$ \\
\hline
\end{tabular}




\begin{tabular}{|c|c|c|c|}
\hline a. Enfoque & b. Alcance & c. Diseño & d. Instrumentos \\
\hline Cuantitativo & $\begin{array}{l}\text { Exploratorio } \\
\text { Descriptivo } \\
\text { Correlacional } \\
\text { Explicativo }\end{array}$ & $\begin{array}{l}\text { - Experimental } \\
\text { - No experimental } \\
\text { - Longitudinal } \\
\text { - No experimental } \\
\text { - Transversal } \\
\text { - Estudio de caso }\end{array}$ & $\begin{array}{l}\text { - Cuestionarios } \\
\text { - Escalas de medición } \\
\text { - Análisis de contenido } \\
\text { cuantitativo } \\
\text { - Pruebas estandarizadas } \\
\text { - Datos secundarios } \\
\text { - Aparatos y equipos } \\
\text { - Indicadores }\end{array}$ \\
\hline Mixto & Todos & Todos & Todos \\
\hline
\end{tabular}

Fuente: elaboración propia.

Nota: * Para llevar a cabo esta caracterización se utilizó la propuesta de Hernández Sampieri, Fernández Collado y Baptista Lucio (2014), quienes definen tipos de alcances, diseños e instrumentos o técnicas de recolección de información.

** Dado que las investigaciones con enfoque cualitativo no siempre delimitan un alcance, se omitió este elemento.

*** El estudio de caso es por naturaleza un diseño metodológico utilizado en el enfoque cualitativo; no obstante, en épocas recientes ha sido usado en las ciencias sociales bajo enfoques de investigación cuantitativos y mixtos. 


\section{Resultados y discusión}

Una vez diligenciada la matriz, se encontró un total de 70 artículos en finanzas bursátiles publicados en las revistas analizadas durante el periodo de estudio.

Figura 1. Número de artículos publicados por año

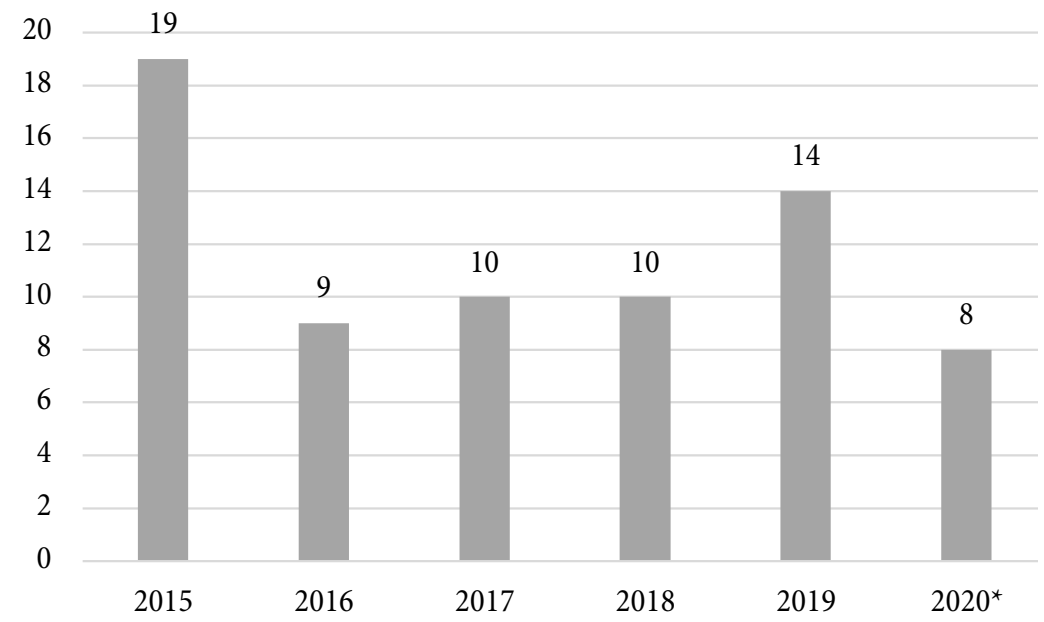

Fuente: elaboración propia.

Nota: ${ }^{\star}$ Del 2020 solo se recolectaron datos hasta el 30 de junio.

En la figura 1 se muestra una evolución año a año de las publicaciones colombianas en el área de finanzas bursátiles. En este sentido, se puede observar que el año en el cuál más artículos se publicaron fue el 2015 con 19 artículos, seguido del 2019 con 14 artículos. El promedio de artículos por año fue de 11.6, por tanto, los años 2016, 2017, 2018 y 2020 están por debajo del promedio. No obstante, vale la pena aclarar que, del último año, solo se incluyeron publicaciones hasta junio de 2020, por tanto, puede que una vez se lleve a cabo el análisis de todo el año, los resultados sean mucho mayores en este caso específico. 
Figura 2. Número de artículos publicados por revista

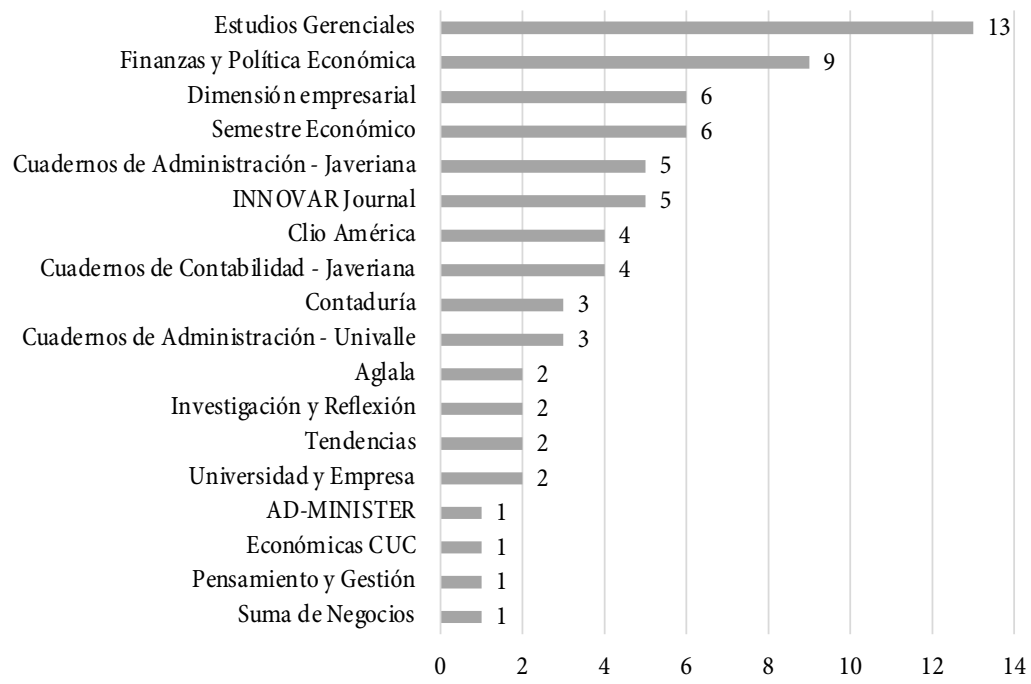

Fuente: elaboración propia.

En la figura 2 se presenta la distribución de artículos de finanzas bursátiles según las revistas en las que fueron publicados. De tal forma, se puede observar que las revistas con mayor participación en esta temática durante los años de estudio fueron Estudios Gerenciales de la Universidad ICESI y Finanzas y Política Económica de la Universidad Católica de Colombia, las cuales, de manera conjunta componen el $31.4 \%$ del total de publicaciones. Mientras que, las revistas con menor número de publicaciones fueron AD-minister de la Universidad EAFIT, Económicas CUC de la Universidad de la Costa, Pensamiento y Gestión de la Universidad del Norte y Suma de Negocios de la Fundación Universitaria Konrad Lorenz, en las cuales únicamente se publicó un artículo durante el periodo de estudio. Cabe aclarar que, en 3 de las 21 revistas analizadas no se encontraron publicaciones de finanzas bursátiles durante el periodo de estudio: Aibi de la Universidad de Santander, Criterio Libre de la Universidad Libre y Revista Escuela de Administración de Negocios de la Universidad EAN. 
Figura 3. Tipología de artículos

80

70

67

60

50

40

30

20

10

0

1

2

Revisión

Reflexión

Investigación

Fuente: elaboración propia.

En la figura 3 se muestra la distribución de los artículos según su tipología. De esta manera, se logra observar que 67 de los 70 artículos publicados son de investigación, lo cual corresponde al $95.71 \%$, mientras que solo se publicaron dos de reflexión y uno de revisión, representando 2.86 \% y $1.43 \%$ respectivamente.

\section{Caracterización de las metodologías de investigación}

Una vez realizada la identificación general de los artículos, se procede a caracterizar su metodología, determinando, como se mencionó anteriormente, el enfoque de investigación, el alcance, el diseño metodológico y los instrumentos o herramientas de recolección de información. Esto se lleva a cabo con la finalidad de conocer cómo se han establecido los diseños metodológicos utilizados en la investigación financiera bursátil en Colombia, para así, contribuir a la caracterización de su estado actual. 
Figura 4. Enfoque de investigación utilizado

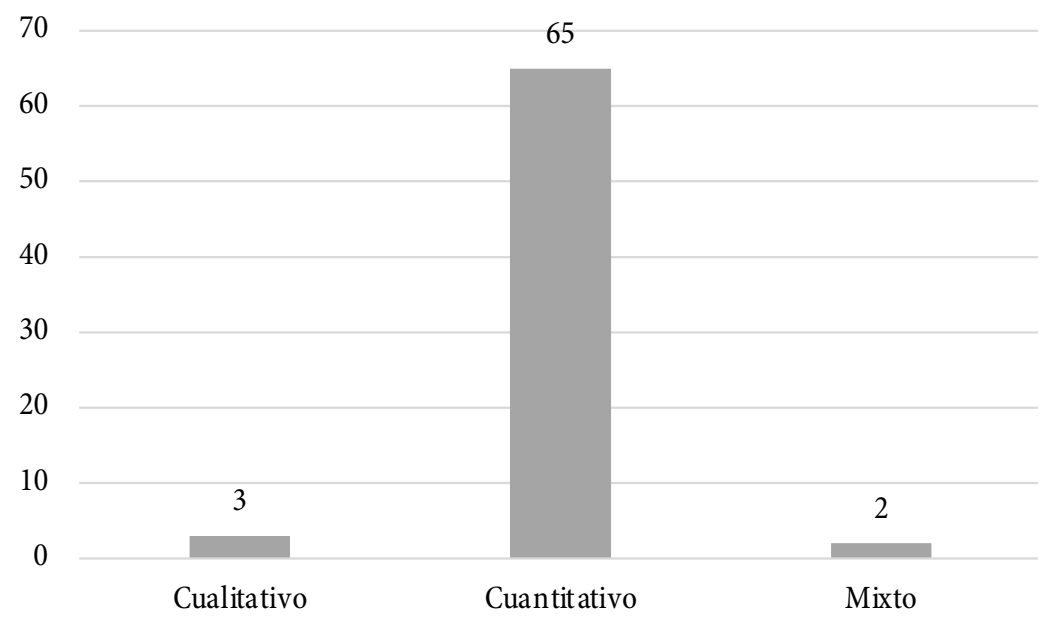

Fuente: elaboración propia.

En la figura 4 se muestra la distribución de los artículos según el enfoque de investigación que en ellos se utilizó. De este modo, se puede observar que en 65 artículos se llevó a cabo la investigación empleando un enfoque cuantitativo, lo cual corresponde al $92.86 \%$ del total. Pero, únicamente tres artículos aplicaron el enfoque cualitativo y dos el mixto, lo que representa $4.29 \%$ y $2.86 \%$ respectivamente. Estos resultados indican que existe una inclinación significativa por parte de los autores por el abordaje de la investigación en finanzas bursátiles desde perspectivas cuantitativas donde prima el análisis numérico y estadístico. En este sentido, es poco lo que se considera desde el punto de vista cualitativo para intentar explicar los fenómenos financieros en los mercados. 
Figura 5. Alcance metodológico según el enfoque

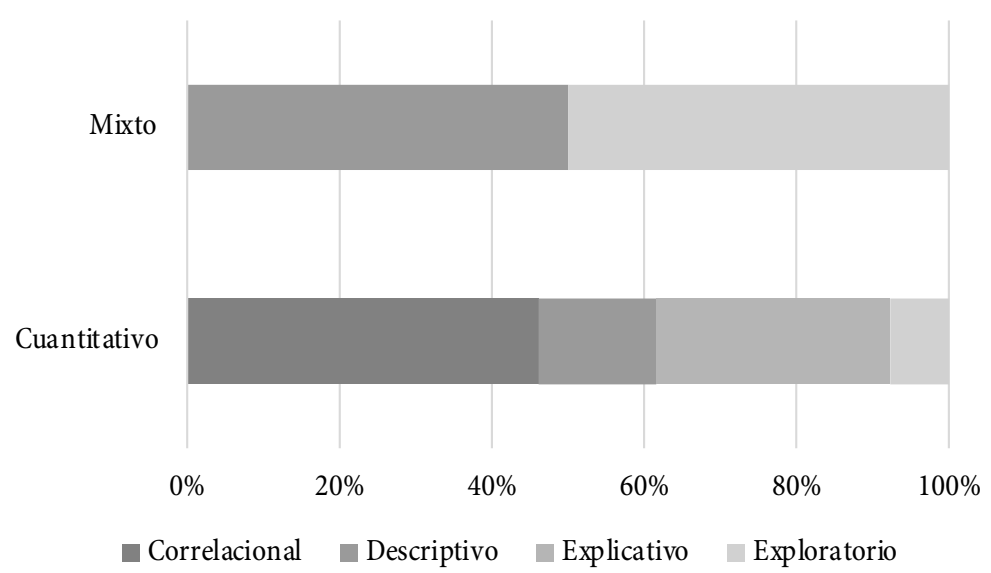

Fuente: elaboración propia.

En la figura 5 se puede observar que, de los dos artículos que utilizaron un enfoque de investigación mixto, el $50 \%$ tuvo un alcance descriptivo (Villanueva García et al., 2015) y el 50 \% restante tuvo un alcance exploratorio (Riascos y Molina Muñoz, 2016). Por otro lado, de los 65 artículos con enfoque de investigación cuantitativo, el $46.15 \%$ tuvo un alcance correlacional, el $30.77 \%$ tuvo un alcance explicativo .

Es evidente que, en los artículos estudiados, el alcance correlacional y el alcance explicativo aplicados en un enfoque cuantitativo fueron predilectos en el periodo de evaluación, representando en conjunto alrededor del $70 \%$ del total de artículos con este enfoque. En este sentido, se podría entender que la mayoría de las investigaciones se han encaminado hacia el uso de herramientas matemáticas, estadísticas y econométricas, bien sea, para intentar generar relaciones de causa-efecto entre fenómenos financieros de los mercados de capitales, o para explicar dichos fenómenos a partir de otras variables. No obstante, es muy poco lo que se hace en términos descriptivos y exploratorios. 
Figura 6. Diseño metodológico según el enfoque

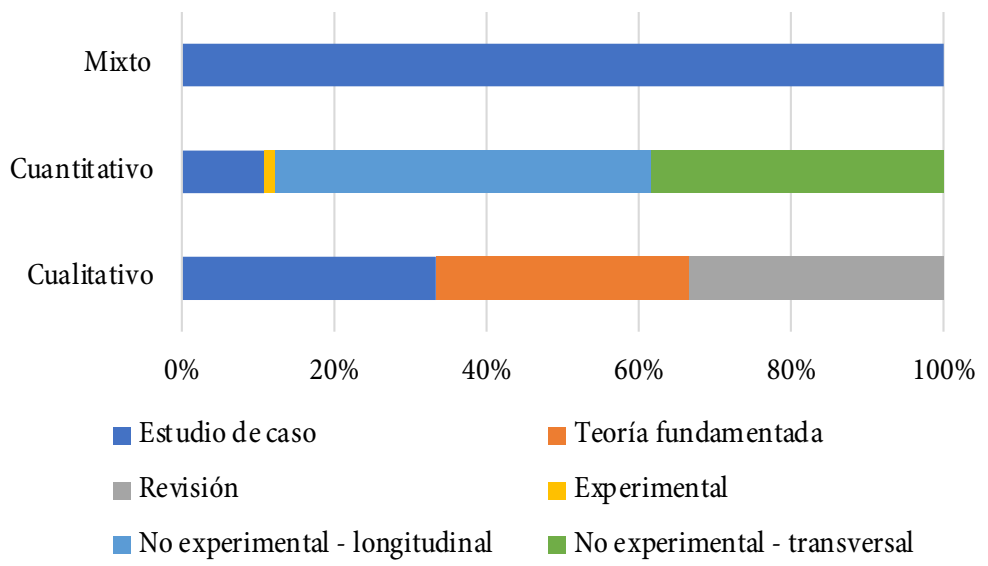

Fuente: elaboración propia.

En la figura 6 se presenta la distribución del diseño metodológico según el enfoque de investigación utilizado. En este sentido, se puede observar que, de los artículos que emplearon un enfoque de investigación mixto, el $100 \%$ lo hizo por medio de estudios de caso (Riascos y Molina Muñoz, 2016; Villanueva García et al., 2015); adicionalmente, de los artículos que utilizaron un enfoque cuantitativo, el 49.23 \% llevó a cabo un diseño no experimental de corte longitudinal y el $1.54 \%$ utilizó un diseño experimental (Peña y Gómez-Mejía, 2019). Finalmente, de los artículos que aplicaron un enfoque de investigación cualitativo, el $33.33 \%$ realizó estudios de caso (Castro Alfaro y Anturi Santos, 2015), otro $33.33 \%$ aplicó teoría fundamentada (Quintero, 2016) y el $33.33 \%$ restante llevó a cabo revisiones de literatura (Useche Arévalo, 2015).

Por un lado, es evidente la primacía de los diseños longitudinales para aquellos artículos que utilizaron un enfoque cuantitativo, dado que tanto los diseños no experimentales como los transversales fueron aplicados repetidamente en las investigaciones estudiadas. Por otro lado, para los trabajos cualitativos es importante notar que ningún artículo utilizó diseños etnográficos, narrativos y de investigación-acción, evidenciando que 
posiblemente existe un campo de estudio en el área financiera bursátil con estas metodologías que no se ha explorado en los últimos años. Asimismo, vale la pena resaltar el uso de los estudios de caso desde enfoques tanto cualitativos, como cuantitativos y mixtos, demostrando que este tipo de diseño probablemente se presta para abordajes metodológicos mucho más amplios en comparación con los demás.

Figura 7. Métodos de recolección de información utilizados según el enfoque

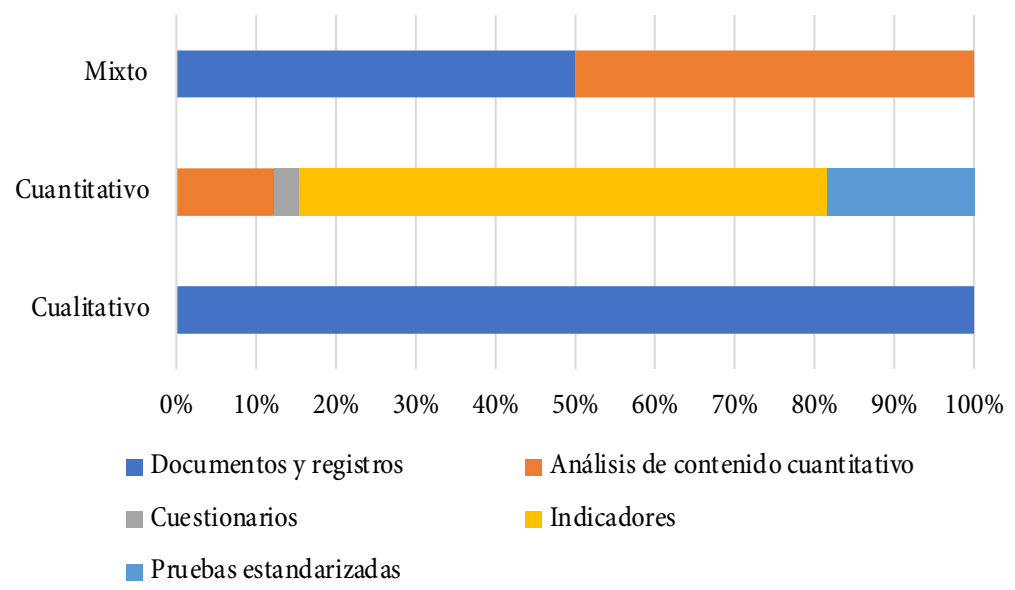

Fuente: elaboración propia.

En la figura 7 se presenta la distribución de los métodos de recolección de información utilizados según el enfoque de investigación. Allí, se puede observar que, de los artículos que emplearon un enfoque de investigación mixto, el $50 \%$ utilizó análisis de contenido cuantitativo (Riascos y Molina Muñoz, 2016) y el 50 \% hicieron uso de documentos y registros (Villanueva García et al., 2015); además, de los artículos que aplicaron un enfoque de investigación cuantitativo, $66.15 \%$ utilizaron indicadores, el $18.46 \%$ utilizaron pruebas estandarizadas, el $12.31 \%$ usaron análisis de contenido cuantitativo y tan solo el $3.08 \%$ aplicaron cuestionarios (Konraht, Lunkes, Gasparetto y Schnorrenberger, 2017; Moreno Alemay y Pereira Villa, 2015). Por último, de los artículos con un enfoque de investigación cualitativo, el 
$100 \%$ hizo uso de documentos y registros como método de recolección de información (Castro Alfaro y Anturi Santos, 2015; Quintero, 2016; Useche Arévalo, 2015).

De acuerdo con lo anterior, se puede resaltar que el uso de indicadores como método de recolección de información para los artículos con enfoque cuantitativo fue el predilecto dentro del periodo de estudio; probablemente, dicho método estuvo asociado con indicadores financieros de empresas, de sectores o de bolsas de valores, con la finalidad de comprender las dinámicas en las cuales se generan estos datos y tratar de generar predicciones a partir de ellos. En contraste, para el enfoque cualitativo únicamente se utilizaron los documentos y registros como método de recolección de información, de nuevo, mostrando que existen amplias posibilidades para abordar la investigación financiera bursátil desde esta perspectiva con el uso de instrumentos como entrevistas, bitácoras, grupos focales o inclusive, la observación.

\section{Conclusiones}

En términos generales, este artículo logra identificar que, para la temática de investigación de las finanzas bursátiles en Colombia durante el periodo de estudio, la metodología de investigación que más se utilizó fue aquella con un enfoque cuantitativo, con alcance correlacional o explicativo, con diseño metodológico longitudinal de corte no experimental o de corte transversal y con métodos de recolección de información basados en indicadores o pruebas estandarizadas. En este sentido, el uso de enfoques de investigación cualitativos o mixtos, con sus respectivos alcances, diseños y métodos es más escaso; esto plantea un vacío en la literatura que, a largo plazo podría explorarse ampliando las posibilidades de investigación enfocadas en explicación de fenómenos sociales y contextuales a través de variables relacionadas con la conducta humana y el comportamiento de los agentes.

Adicionalmente, conviene cuestionarse sobre los motivos para los cambios significativos en el número de publicaciones que han surgido a través 
del tiempo, su distribución en las revistas seleccionadas para este estudio y los tipos de artículos escritos y publicados. En principio, el decrecimiento inicial que tuvo el número de artículos entre 2015 y 2016 es un punto de inflexión que requiere de un mayor análisis y que plantea desafíos para la academia en términos de incrementar nuevamente las publicaciones o, al menos, de conocer las causas de esta disminución.

Asimismo, la distribución desigual entre las revistas hace pensar que algunas de ellas tienen mayor acogida para estos temas, ya sea porque los autores las consideran más afines a ellos o porque los comités editoriales tienen criterios de recepción y evaluación que favorecen la temática particular; no obstante, convendría profundizar en este fenómeno para conocer a profundidad las condiciones específicas que lo originan. Por último, dado que la gran mayoría de artículos han sido de investigación, hay un amplio campo por explorar en cuanto a las revisiones de literatura utilizando metodologías rigurosas (como la revisión sistemática) o, inclusive, respecto a las reflexiones sobre las finanzas bursátiles que permitan repensar la manera de investigar en esta área y el contexto en el que se desenvuelven las empresas, los agentes y los mercados.

Finalmente, en términos de las metodologías aplicadas es notorio que la cuantitativas priman; sin embargo, todavía existen posibilidades de indagación en este tipo de enfoque, utilizando alcances exploratorios o descriptivos, diseños experimentales o estudios de caso, e instrumentos como cuestionarios, escalas de medición, análisis de contenido cuantitativo, datos secundarios o aparatos y equipos. Además de esto, en términos de enfoques cualitativos y mixtos, las oportunidades de exploración a futuro en la literatura son aún más amplias, dado que poco se ha investigado utilizando este enfoque en el área y sus bondades, en términos de conocer desde perspectivas sociales y contextuales las condiciones que subyacen los fenómenos financieros pueden traer grandes beneficios para el avance académico de las finanzas bursátiles. 


\section{Referencias}

Améstica Rivas, L. R., Campos Fuentes, D. A., y Cornejo-Saavedra, E. E. (2017). Mergers and acquisitions announcements and effects on stock returns: Chile, 2010-2014. Cuadernos de Administración, 30(54), 39-64. https://doi. org/10.11144/javeriana.cao30-54.afae

Atehortúa Granados, J. A. (2012). Mercado de capitales y portafolios de inversión. Sello Editorial de la Universidad de Medellín.

Cárdenas, M., y Rojas, J. (1996). El mercado accionario en la economía colombiana. En M. Cárdenas, y J. Rojas, Misión de estudios del mercado de capitales. https://www.repository.fedesarrollo.org.co/bitstream/handle/11445/1509/ Repor_Febrero_1996_Cardenas_y_Rojas.pdf?sequence=3\&isAllowed=y

Castro Alfaro, A., y Anturi Santos, R. (2015). El análisis técnico y fundamental en un contexto de globalización: Bancolombia The technical and fundamental analysis in a context of globalization: Bancolombia. Aglala, 6(73601), 1-39. https://doi.org/10.22519/22157360.976

Hernández Sampieri, R., Fernández Collado, C., y Baptista Lucio, P. (2014). Metodología de la investigación (vol. 6). Mcgraw-hill.

Konraht, J., Lunkes, R., Gasparetto, V., y Schnorrenberger, D. (2017). Remuneração Variável: Um Estudo da Relação com Indicadores Financeiros das Companhias Abertas Brasileiras. Revista Facultad de Ciencias Económicas, 26(1), 209-224. https://doi.org/10.18359/rfce.1781

Moreno Alemay, P., y Pereira Villa, C. (2015). Why does Colombia lack agricultural commodity futures?. Revista Finanzas y Política Económica, 7(2), 325-339. https://doi.org/10.14718/revfinanzpolitecon.2015.7.2.6

Peña, V. A., y Gómez-Mejía, A. (2019). Effect of heuristic anchoring and adjustment, and optimism bias, in stock market forecasts. Revista Finanzas y Política Económica, 11(2), 389-409. https://doi.org/10.14718/revfinanzpolitecon.2019.11.2.10

Riascos, J. C., y Molina Muñoz, J. E. (2016). Breves consideraciones acerca de la importancia de los árboles de decisión en el análisis de carteras. Tendencias, 17(1), 11. https://doi.org/10.22267/rtend.161701.11

Uribe Gil, J. M. (2007). Caracterización del mercado accionario colombiano, 20012006: un análisis comparativo. Borradores de Economía, 456. 
Useche Arévalo, A. J. (2015). Construcción de portafolios de inversión desde las finanzas del comportamiento: una revisión crítica. Cuadernos de Administración, 28(51), 11-43. https://doi.org/10.11144/Javeriana.cao28-51.cpif

Vera Leyton, M. (2020). Contagion of the shareholder market: cases of Colombia, México, Perú, Chile and Argentina. Dimensión Empresarial, 18(1). https://doi. org/10.15665/dem.v18i(1).2068

Villanueva García, J., Zorio Grima, A., y García Benau, M. A. (2015). Supervisores bursátiles, gobierno corporativo y cumplimiento de las NIIF: el caso de la CNMV. Innovar, 25(55), 89-100. https://doi.org/10.15446/innovar.v25n55.47198 